\title{
The influence of storage on the human milk lipidome stability for the lipidomic studies - Supplementary materials
}

Dorota Garwolińska ${ }^{1}$, Michał Młynarczykㄹ, Agata Kot-Wasik , and Weronika HeweltBelka ${ }^{1, *}$

'Department of Analytical Chemistry, Faculty of Chemistry, Gdańsk University of Technology, Gabriela Narutowicza 11/12, 80-233 Gdańsk, Poland

Corresponding Author

*e-mail: werbelka@pg.edu.pl.

Table of contents

\begin{tabular}{|c|c|c|}
\hline & Supporting Information & Page \\
\hline & Reagents and materials. & S-2 \\
\hline & Sample preparation & S-2 \\
\hline & LC-MS analysis. & S-2 \\
\hline & Data treatment. & S-2 \\
\hline Table S1 & $\begin{array}{l}\text { The \%RSD values calculated for TG relative amounts among all samples } \\
\text { stored at }-20^{\circ} \mathrm{C} \text { and }-80^{\circ} \mathrm{C}\end{array}$ & S-3 \\
\hline Table S2 & $\begin{array}{l}\text { The \%RSD values calculated for DG peak volumes detected among all } \\
\text { samples stored at }-20^{\circ} \mathrm{C} \text { and }-80^{\circ} \mathrm{C}\end{array}$ & S-4 \\
\hline Figure S1 & $\begin{array}{l}\text { The PCA of the TG profiles (\%relative amount) of HM samples stored at (A) } \\
-20^{\circ} \mathrm{C} \text { and (B) }-80^{\circ} \mathrm{C} \text { and collected from individual women: W13A (yellow } \\
\text { circle); W8 (pink circles); W18 (green circles); W13B (blue circles); W4 } \\
\text { (grey circles). }\end{array}$ & S-4 \\
\hline Table S3 & $\begin{array}{l}\text { TGs statistically significantly different between the individual mothers } \\
\text { accordingly to the Mann-Whitney test unpaired }(p<0.05) \text {. }\end{array}$ & S-5 \\
\hline Table S4 & $\begin{array}{l}\text { Phospholipids statistically significantly different between the individual } \\
\text { mothers accordingly to the Mann-Whitney test unpaired }(p<0.05) \text {. }\end{array}$ & S-6 \\
\hline Figure S2 & $\begin{array}{l}\text { PCA of the phospholipids profiles of } \mathrm{HM} \text { samples stored at (A) }-20^{\circ} \mathrm{C} \text { and } \\
\text { (B) }-80^{\circ} \mathrm{C} \text { and collected from particular women: W13A (yellow circle); W8 } \\
\text { (pink circles); W18 (green circles); W13B (blue circles); W4 (grey circles). }\end{array}$ & S-6 \\
\hline Table S5 & $\begin{array}{l}\text { The relative amounts of TGs detected in samples that underwent up to } \\
\text { three freeze-thawed cycles ( } 1 \mathrm{FT} \text { - one freeze-thaw cycle, } 2 \mathrm{FT} \text { - two freeze- } \\
\text { thaw cycles, } 3 \text { - three freeze-thaw cycles) }\end{array}$ & S-7 \\
\hline Table S6 & $\begin{array}{l}\text { The peak volumes of DGs detected in samples that underwent up to three } \\
\text { freeze-thawed cycles (1FT - one freeze-thaw cycle, } 2 \mathrm{FT}-\text { two freeze-thaw } \\
\text { cycles, } 3 \text { - three freeze-thaw cycles) }\end{array}$ & S-8 \\
\hline Table S7 & $\begin{array}{l}\text { The \%RSD values calculated for phospholipid relative amounts in samples } \\
\text { that underwent three freeze-thawed cycles }\end{array}$ & S-9 \\
\hline
\end{tabular}


Reagents and materials. LC-MS-grade methanol, HPLC-grade chloroform and n-hexane were purchased from Merck (Darmstadt, Germany). HPLC-grade 2-propanol, ammonium formate $(99.9 \%$ purity), formic acid and ammonia p.a. were purchased from Sigma-Aldrich (St. Louis, MO, USA). Deionized water was purified on an HLP5 system (Hydrolab, Wislina, Poland).

Sample preparation. After 10 s of vigorous vortexing, $100 \mu \mathrm{L} \mathrm{HM}$ sample was transferred and mixed with $900 \mu \mathrm{L} 1 \%$ formic acid in methanol in a polypropylene tube. Next, sample was vortex for $30 \mathrm{~s}$, and centrifuge for $10 \mathrm{~min}$ at $10,732 \times \mathrm{g}$. The $900 \mu \mathrm{L}$ of obtained supernatant was transferred to a HybridSPE-Phospholipid (bed weight $20 \mathrm{mg}$ ) cartridge (Supelco, Sigma Aldrich, St. Louis, MO, USA). After supernatant loading, the stationary phase was washed with the use of methanol and 2-propanol, and next phospholipids were eluted with $2 \mathrm{~mL} 5 \%$ ammonia in methanol. The obtained phospholipid extract was evaporated to dryness under a nitrogen stream and dissolved in the 50-fold diluted extract obtained in the LLE-based step. LLE-based step was performed based on modified Bligh and Dyer extraction. After $10 \mathrm{~s}$ of vigorous vortexing, $225 \mu \mathrm{L} \mathrm{HM}$ sample was transferred and mixed with $950 \mu \mathrm{L}$ chloro-form/methanol $(1 / 2, v / v)$ in a borosilicate glass tube with a PTFE cap. After $10 \mathrm{~s}$ of vortexing, $310 \mu \mathrm{L}$ chloroform and $310 \mu \mathrm{L}$ water were added. To separate the organic phases containing lipids, the sample was centrifuged for $10 \mathrm{~min}$ at $5000 \times \mathrm{g}$. The $20 \mu \mathrm{L}$ lower organic fraction was transferred to glass test tube and mix with $980 \mu \mathrm{L}$ methanol containing IS $(1 \mu \mathrm{g} / \mathrm{mL}, \mathrm{PC} 18: 0 / 18: 0))$. The $100 \mu \mathrm{L}$ prepared diluted lipid extract was used to dissolve phospholipid extract evaporated to dryness in previous SPE-based step. The obtained enriched-diluted extract was transferred to a chromatographic vial and analyzed by RP-LC-Q-TOF-MS.

LC-MS analysis. Briefly, lipids were chromatography separated on a on a reversed-phase column (Poroshell $120 \mathrm{EC}-\mathrm{C} 8,2.1 \times 150 \mathrm{~mm}, 1.9 \mu \mathrm{m}$ particle size, Agilent) with a $0.2 \mu \mathrm{m}$ in-line filter. The column was maintained at $45^{\circ} \mathrm{C}$. The mobile phase consisted of a component $A$ : a mixture of $5 \mathrm{mM}$ ammonium formate in water and methanol $(1 / 4, v / v)$ and component $B$ : a mixture of $5 \mathrm{mM}$ ammonium formate in water, $\mathrm{n}$-hexane and 2-propanol $(1 / 20 / 79, \mathrm{v} / \mathrm{v} / \mathrm{v})$ and was pumped with a flow rate 0.5 $\mathrm{mL} / \mathrm{min}$ using gradient program. Total run time was $30.5 \mathrm{~min}$, and the injection volume was $0.5 \mu \mathrm{L}$.

The MS data were collected in positive ion mode using the SCAN acquisition mode in a range from 200 to $1700 \mathrm{~m} / \mathrm{z}$ in high-resolution mode $(4 \mathrm{GHz})$. The remaining MS parameters were set as follows: capillary voltage of $3500 \mathrm{~V}$ and fragmentor voltage of $120 \mathrm{~V}$, nebulizer gas pressure of $35 \mathrm{psi}$, drying gas flow rate of $10 \mathrm{~L} / \mathrm{min}$ and temperature of $300^{\circ} \mathrm{C}$.

Data treatment. The Batch Targeted Feature Extraction algorithm was performed with the following parameters: positive ions, charge carriers- $\mathrm{H}+, \mathrm{Na}+, \mathrm{NH} 4+$; match tolerance, $15 \mathrm{ppm}$; retention time, $0.3 \mathrm{~min}$; Gaussian smoothing before extracted ion chromatogram extraction (EIC) filtering on peak height, 1000 counts. The .cef files were ex-ported and imported to Mass Profiler Professional 15.1 software (Agilent Technologies, Santa Clara, CA, USA) for data alignment and filtration. Missing values were exported as missing. The alignment parameters were set as follows: alignment slope = $0.0 \%$; alignment intercept $=0.4 \mathrm{~min}$; mass tolerance slope $=20.0 \mathrm{ppm}$; intercept $=2.0 \mathrm{mDa}$. Filtration was based on the peak characteristics (peak symmetry, tailing), frequency (the MFs remained in the dataset if they were present in $100 \%$ of the samples in at least one specified group (specific storage condition)) and the QC \%RSD (MFs remained if \%RSD $<30 \%$ in all the QC samples). The MFs that were present in the extraction blank, with the average peak volume higher than $10 \%$ of the average peak volume in the real samples, were removed. 
Table S2. The \%RSD values calculated for TG relative amounts among all samples stored at $-20^{\circ} \mathrm{C}$ and $-80^{\circ} \mathrm{C}$

\begin{tabular}{|c|c|c|c|c|c|c|c|c|c|c|}
\hline \multirow[b]{2}{*}{ Compound } & \multicolumn{5}{|c|}{ storing at- $20^{\circ} \mathrm{C}$} & \multicolumn{5}{|c|}{ storing at $-80^{\circ} \mathrm{C}$} \\
\hline & W13A & W8 & W18 & W13B & W4 & W13A & W8 & W18 & W13B & W4 \\
\hline TG30:0 & $26 \%$ & $13 \%$ & $13 \%$ & $24 \%$ & $16 \%$ & $9 \%$ & $17 \%$ & $20 \%$ & $11 \%$ & $13 \%$ \\
\hline TG34:1 & $12 \%$ & $9 \%$ & $7 \%$ & $10 \%$ & $13 \%$ & $12 \%$ & $13 \%$ & $17 \%$ & $11 \%$ & $14 \%$ \\
\hline TG36:0 & $22 \%$ & $15 \%$ & $14 \%$ & $16 \%$ & $20 \%$ & $8 \%$ & $10 \%$ & $14 \%$ & $12 \%$ & $9 \%$ \\
\hline TG36:1 & $13 \%$ & $5 \%$ & $10 \%$ & $12 \%$ & $12 \%$ & $11 \%$ & $11 \%$ & $15 \%$ & $12 \%$ & $11 \%$ \\
\hline TG38:0 & $10 \%$ & $5 \%$ & $7 \%$ & $17 \%$ & $13 \%$ & $10 \%$ & $8 \%$ & $12 \%$ & $11 \%$ & $9 \%$ \\
\hline TG38:1 & $11 \%$ & $5 \%$ & $11 \%$ & $10 \%$ & $13 \%$ & $9 \%$ & $11 \%$ & $11 \%$ & $9 \%$ & $10 \%$ \\
\hline TG38:2 & $5 \%$ & $10 \%$ & $7 \%$ & $2 \%$ & $15 \%$ & $13 \%$ & $12 \%$ & $18 \%$ & $14 \%$ & $13 \%$ \\
\hline TG40:0 & $9 \%$ & $15 \%$ & $9 \%$ & $10 \%$ & $10 \%$ & $18 \%$ & $12 \%$ & $15 \%$ & $8 \%$ & $11 \%$ \\
\hline TG40:1 & $7 \%$ & $8 \%$ & $5 \%$ & $8 \%$ & $12 \%$ & $5 \%$ & $6 \%$ & $8 \%$ & $8 \%$ & $7 \%$ \\
\hline TG42:1 & $9 \%$ & $9 \%$ & $4 \%$ & $9 \%$ & $13 \%$ & $3 \%$ & $5 \%$ & $4 \%$ & $7 \%$ & $6 \%$ \\
\hline TG44:1 & $4 \%$ & $4 \%$ & $3 \%$ & $1 \%$ & $1 \%$ & $8 \%$ & $5 \%$ & $4 \%$ & $5 \%$ & $5 \%$ \\
\hline TG44:2 & $8 \%$ & $9 \%$ & $3 \%$ & $7 \%$ & $10 \%$ & $4 \%$ & $3 \%$ & $3 \%$ & $6 \%$ & $4 \%$ \\
\hline TG46:1 & $8 \%$ & $8 \%$ & $8 \%$ & $8 \%$ & $2 \%$ & $7 \%$ & $6 \%$ & $5 \%$ & $4 \%$ & $5 \%$ \\
\hline TG46:2 & $4 \%$ & $3 \%$ & $6 \%$ & $5 \%$ & $4 \%$ & $7 \%$ & $3 \%$ & $4 \%$ & $7 \%$ & $6 \%$ \\
\hline TG46:3 & $6 \%$ & $7 \%$ & $2 \%$ & $8 \%$ & $8 \%$ & $3 \%$ & $6 \%$ & $4 \%$ & $4 \%$ & $4 \%$ \\
\hline TG48:2 & $5 \%$ & $4 \%$ & $9 \%$ & $4 \%$ & $3 \%$ & $4 \%$ & $3 \%$ & $6 \%$ & $5 \%$ & $4 \%$ \\
\hline TG48:3 & $4 \%$ & $5 \%$ & $2 \%$ & $4 \%$ & $2 \%$ & $6 \%$ & $7 \%$ & $1 \%$ & $5 \%$ & $5 \%$ \\
\hline TG48:4 & $3 \%$ & $7 \%$ & $4 \%$ & $5 \%$ & $5 \%$ & $6 \%$ & $4 \%$ & $6 \%$ & $3 \%$ & $5 \%$ \\
\hline TG50:2 & $5 \%$ & $5 \%$ & $7 \%$ & $5 \%$ & $6 \%$ & $9 \%$ & $6 \%$ & $4 \%$ & $6 \%$ & $8 \%$ \\
\hline TG50:3 & $6 \%$ & $2 \%$ & $4 \%$ & $6 \%$ & $4 \%$ & $8 \%$ & $7 \%$ & $6 \%$ & $3 \%$ & $6 \%$ \\
\hline TG50:4 & $2 \%$ & $5 \%$ & $3 \%$ & $4 \%$ & $7 \%$ & $8 \%$ & $10 \%$ & $2 \%$ & $2 \%$ & $4 \%$ \\
\hline TG52:2 & $3 \%$ & $6 \%$ & $5 \%$ & $2 \%$ & $2 \%$ & $6 \%$ & $6 \%$ & $5 \%$ & $5 \%$ & $3 \%$ \\
\hline TG52:3 & $4 \%$ & $2 \%$ & $4 \%$ & $2 \%$ & $4 \%$ & $3 \%$ & $3 \%$ & $9 \%$ & $2 \%$ & $6 \%$ \\
\hline TG52:4 & $7 \%$ & $6 \%$ & $4 \%$ & $7 \%$ & $6 \%$ & $8 \%$ & $6 \%$ & $1 \%$ & $4 \%$ & $4 \%$ \\
\hline TG54:2 & $14 \%$ & $15 \%$ & $20 \%$ & $7 \%$ & $11 \%$ & $18 \%$ & $12 \%$ & $15 \%$ & $8 \%$ & $7 \%$ \\
\hline TG54:3 & $8 \%$ & $9 \%$ & $9 \%$ & $9 \%$ & $7 \%$ & $10 \%$ & $11 \%$ & $6 \%$ & $5 \%$ & $6 \%$ \\
\hline TG54:4 & $7 \%$ & $7 \%$ & $5 \%$ & $7 \%$ & $7 \%$ & $9 \%$ & $3 \%$ & $4 \%$ & $3 \%$ & $7 \%$ \\
\hline
\end{tabular}


Table S2. The \%RSD values calculated for DG peak volumes detected among all samples stored at $-20^{\circ} \mathrm{C}$ and $80^{\circ} \mathrm{C}$

\begin{tabular}{lcccccccccc}
\hline & \multicolumn{3}{c}{ storing at-20 ${ }^{\circ} \mathrm{C}$} & \multicolumn{5}{c}{ storing at -80 ${ }^{\circ} \mathrm{C}$} \\
Compound & W13A & W8 & W18 & W13B & W4 & W13A & W8 & W18 & W13B & W4 \\
\hline DG24:0 & $14 \%$ & $13 \%$ & $11 \%$ & $13 \%$ & $10 \%$ & $20 \%$ & $6 \%$ & $9 \%$ & $13 \%$ & $14 \%$ \\
DG 26:0 & $13 \%$ & $8 \%$ & $8 \%$ & $6 \%$ & $10 \%$ & $12 \%$ & $8 \%$ & $7 \%$ & $8 \%$ & $12 \%$ \\
DG 28:0 & $6 \%$ & $4 \%$ & $4 \%$ & $5 \%$ & $9 \%$ & $11 \%$ & $7 \%$ & $9 \%$ & $8 \%$ & $11 \%$ \\
DG 28:1 & $13 \%$ & $7 \%$ & $9 \%$ & $15 \%$ & $14 \%$ & $15 \%$ & $13 \%$ & $21 \%$ & $15 \%$ & $10 \%$ \\
DG30:0 & $13 \%$ & $8 \%$ & $9 \%$ & $7 \%$ & $9 \%$ & $15 \%$ & $6 \%$ & $4 \%$ & $11 \%$ & $16 \%$ \\
DG30:1 & $3 \%$ & $6 \%$ & $3 \%$ & $3 \%$ & $12 \%$ & $4 \%$ & $9 \%$ & $9 \%$ & $8 \%$ & $6 \%$ \\
DG30:2 & $8 \%$ & $7 \%$ & $3 \%$ & $10 \%$ & $19 \%$ & $7 \%$ & $14 \%$ & $10 \%$ & $13 \%$ & $6 \%$ \\
DG32:1 & $5 \%$ & $4 \%$ & $5 \%$ & $7 \%$ & $8 \%$ & $5 \%$ & $5 \%$ & $2 \%$ & $3 \%$ & $4 \%$ \\
DG32:2 & $2 \%$ & $7 \%$ & $4 \%$ & $4 \%$ & $19 \%$ & $12 \%$ & $8 \%$ & $5 \%$ & $7 \%$ & $10 \%$ \\
DG34:1 & $1 \%$ & $3 \%$ & $5 \%$ & $4 \%$ & $8 \%$ & $3 \%$ & $3 \%$ & $4 \%$ & $3 \%$ & $4 \%$ \\
DG34:2 & $4 \%$ & $2 \%$ & $4 \%$ & $3 \%$ & $7 \%$ & $3 \%$ & $4 \%$ & $3 \%$ & $2 \%$ & $3 \%$ \\
DG34:3 & $4 \%$ & $13 \%$ & $18 \%$ & $10 \%$ & $21 \%$ & $7 \%$ & $7 \%$ & $5 \%$ & $5 \%$ & $4 \%$ \\
DG36:2 & $12 \%$ & $8 \%$ & $9 \%$ & $7 \%$ & $17 \%$ & $17 \%$ & $10 \%$ & $15 \%$ & $14 \%$ & $16 \%$ \\
DG36:3 & $7 \%$ & $3 \%$ & $8 \%$ & $5 \%$ & $14 \%$ & $4 \%$ & $6 \%$ & $3 \%$ & $8 \%$ & $6 \%$ \\
DG36:4 & $13 \%$ & $5 \%$ & $6 \%$ & $10 \%$ & $15 \%$ & $17 \%$ & $4 \%$ & $4 \%$ & $4 \%$ & $11 \%$ \\
\hline
\end{tabular}

(A)

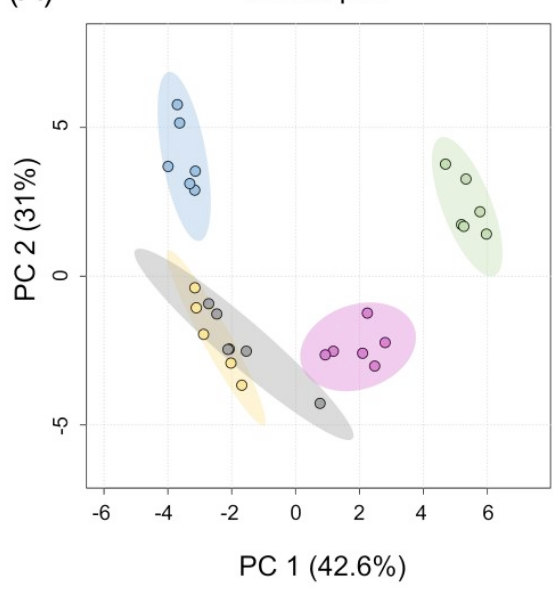

(B)

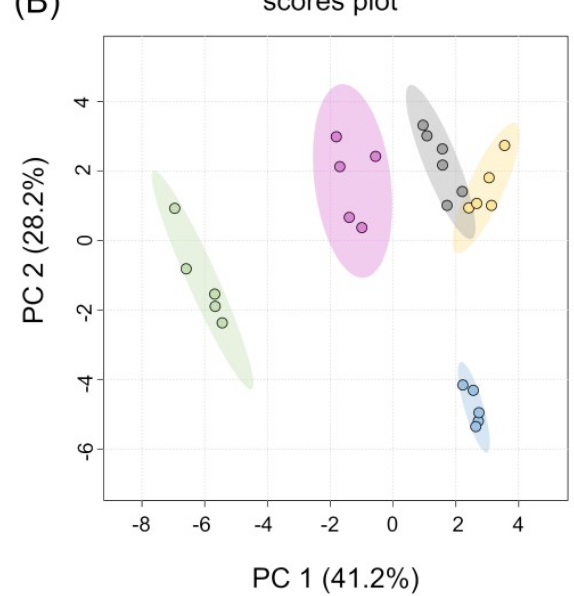

OW13A OW8 OW18 OW13B OW4

Figure S1. The PCA of the TG profiles (\%relative amount) of $\mathrm{HM}$ samples stored at $(\mathrm{A})-20^{\circ} \mathrm{C}$ and (B) $-80^{\circ} \mathrm{C}$ and collected from individual women: W13A (yellow circle); W8 (pink circles); W18 (green circles); W13B (blue circles); W4 (grey circles). 
Table S3. TGs statistically significantly different between the individual mothers accordingly to the Mann-Whitney test unpaired $(p<0.05)$.

\begin{tabular}{ccc}
\hline & \multicolumn{2}{c}{$p$-value } \\
compound & storing at-20 ${ }^{\circ} \mathrm{C}$ & storing at $-80^{\circ} \mathrm{C}$ \\
\hline TG30:0 & $2.71 \mathrm{E}-04$ & $7.31 \mathrm{E}-04$ \\
TG34:1 & $1.64 \mathrm{E}-03$ & $7.07 \mathrm{E}-03$ \\
TG36:0 & $1.39 \mathrm{E}-03$ & $5.55 \mathrm{E}-04$ \\
TG36:1 & $4.92 \mathrm{E}-03$ & not significant \\
TG38:0 & $2.19 \mathrm{E}-04$ & $6.85 \mathrm{E}-04$ \\
TG38:1 & $2.62 \mathrm{E}-03$ & $9.51 \mathrm{E}-03$ \\
TG38:2 & $9.65 \mathrm{E}-04$ & $6.80 \mathrm{E}-03$ \\
TG40:0 & $1.58 \mathrm{E}-04$ & $1.57 \mathrm{E}-03$ \\
TG40:1 & $2.11 \mathrm{E}-04$ & $7.56 \mathrm{E}-04$ \\
TG42:1 & $1.84 \mathrm{E}-04$ & $4.44 \mathrm{E}-04$ \\
TG44:1 & $6.90 \mathrm{E}-05$ & $1.24 \mathrm{E}-03$ \\
TG44:2 & $6.24 \mathrm{E}-05$ & $1.46 \mathrm{E}-04$ \\
TG46:1 & $1.75 \mathrm{E}-04$ & $1.30 \mathrm{E}-04$ \\
TG46:2 & $5.88 \mathrm{E}-05$ & $1.85 \mathrm{E}-04$ \\
TG46:3 & $7.87 \mathrm{E}-05$ & $1.67 \mathrm{E}-04$ \\
TG48:2 & $4.49 \mathrm{E}-04$ & $3.36 \mathrm{E}-04$ \\
TG48:3 & $3.41 \mathrm{E}-05$ & $1.37 \mathrm{E}-04$ \\
TG48:4 & $3.50 \mathrm{E}-05$ & $1.20 \mathrm{E}-04$ \\
TG50:2 & $2.07 \mathrm{E}-04$ & $2.00 \mathrm{E}-03$ \\
TG50:3 & $1.13 \mathrm{E}-04$ & $3.19 \mathrm{E}-04$ \\
TG50:4 & $2.25 \mathrm{E}-05$ & $9.16 \mathrm{E}-05$ \\
TG52:2 & $2.23 \mathrm{E}-05$ & $3.05 \mathrm{E}-04$ \\
TG52:3 & $1.00 \mathrm{E}-04$ & $2.14 \mathrm{E}-04$ \\
TG52:4 & $1.55 \mathrm{E}-04$ & $2.07 \mathrm{E}-04$ \\
TG54:2 & $2.25 \mathrm{E}-04$ & $5.49 \mathrm{E}-04$ \\
TG54:3 & $6.16 \mathrm{E}-05$ & $1.78 \mathrm{E}-04$ \\
TG54:4 & $4.28 \mathrm{E}-05$ & $2.11 \mathrm{E}-04$ \\
TG54:5 & $7.61 \mathrm{E}-05$ & $2.14 \mathrm{E}-04$ \\
\hline & &
\end{tabular}


Table S4. Phospholipids statistically significantly different between the individual mothers accordingly to the Mann-Whitney test unpaired $(p<0.05)$.

\begin{tabular}{ccc}
\hline & \multicolumn{2}{c}{$p$-value } \\
compound & storing at-20 ${ }^{\circ} \mathrm{C}$ & storing at $-80^{\circ} \mathrm{C}$ \\
\hline LysoPC16:0 & 0.000257 & 0.000162 \\
Lyso-PC18:0 & 0.000741 & 0.000216 \\
Lyso-PC18:1 & $7.09 \mathrm{E}-05$ & 0.000113 \\
Lyso-PC18:2 & $3.48 \mathrm{E}-05$ & $7.56 \mathrm{E}-05$ \\
Lyso-PE18:1 & $4.69 \mathrm{E}-05$ & $5.38 \mathrm{E}-05$ \\
LYsoPE18:2 & $4.69 \mathrm{E}-05$ & $5.38 \mathrm{E}-05$ \\
PC32:0 & $5.85 \mathrm{E}-05$ & 0.02481 \\
PC34:1 & $5.89 \mathrm{E}-05$ & $3.11 \mathrm{E}-05$ \\
PC34:2 & $3.13 \mathrm{E}-05$ & $5.1 \mathrm{E}-05$ \\
PC36:2 & $5.51 \mathrm{E}-05$ & $2.89 \mathrm{E}-05$ \\
PE34:1 & $2.53 \mathrm{E}-05$ & $2.95 \mathrm{E}-05$ \\
PE34:2 & 0.000106 & 0.000102 \\
PE36:1 & 0.000176 & 0.000154 \\
PE36:2 & $8.29 \mathrm{E}-05$ & $2.2 \mathrm{E}-05$ \\
SMd34:1 & 0.008044 & 0.006134 \\
SMd36:1 & $1.99 \mathrm{E}-05$ & 0.000455 \\
SMd38:1 & 0.019059 & 0.003107 \\
SMd40:1 & 0.001714 & 0.020512 \\
SMd42:1 & 0.000802 & 0.015676 \\
SMd42:2 & $3.1 \mathrm{E}-05$ & $4.84 \mathrm{E}-05$ \\
\hline
\end{tabular}

(A)

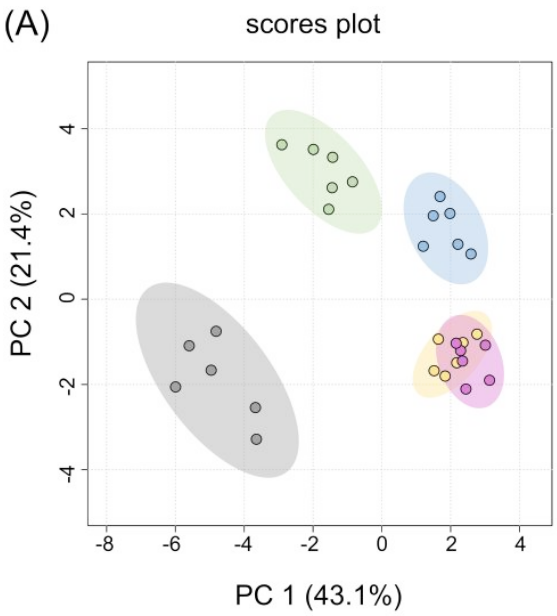

(B) scores plot

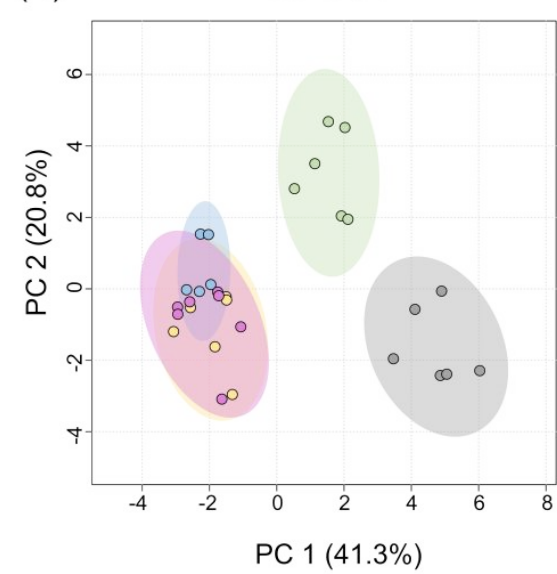

OW13A OW8 OW18 OW13B OW4

Figure S2. PCA of the phospholipids profiles of $H M$ samples stored at $(A)-20^{\circ} \mathrm{C}$ and $(B)-80^{\circ} \mathrm{C}$ and collected from particular women: W13A (yellow circle); W8 (pink circles); W18 (green circles); W13B (blue circles); W4 (grey circles). 


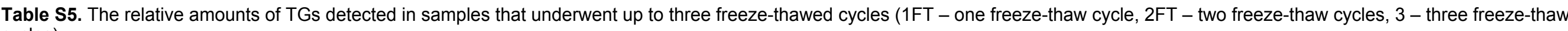
cycles)

\begin{tabular}{|c|c|c|c|c|c|c|c|c|c|c|c|c|c|c|c|}
\hline \multirow[b]{2}{*}{ Compound } & \multicolumn{3}{|c|}{ W13A } & \multicolumn{3}{|c|}{ W8 } & \multicolumn{3}{|c|}{ W18 } & \multicolumn{3}{|c|}{ W1B } & \multicolumn{3}{|c|}{ W4 } \\
\hline & $1 \mathrm{FT}$ & $2 \mathrm{FT}$ & $3 F T$ & $1 \mathrm{FT}$ & $2 \mathrm{FT}$ & $\mathrm{ET}$ & $=\mathrm{T}$ & $2 \mathrm{FT}$ & $3 \mathrm{FT}$ & $\mathrm{FT}$ & $2 \mathrm{FT}$ & $\mathrm{FT}$ & $1 \mathrm{FT}$ & $2 \mathrm{FT}$ & $3 F T$ \\
\hline$: 1$ & 0.1 & 6 & & & & & & & & & & & & & \\
\hline & & & & & & & & & & & & & & & $.64 \%$ \\
\hline & & & & & & & & $0.33 \%$ & & & $0.34 \%$ & & & & $0.22 \%$ \\
\hline TG38:0 & $1.11 \%$ & 1.03 & $0.93 \%$ & $1.12 \%$ & 1.4 & $0.83 \%$ & $1.70 \%$ & $1.63 \%$ & $63 \%$ & & & & & & $97 \%$ \\
\hline & & & & & & & & & & & & & & & $77 \%$ \\
\hline TG38:2 & $0.31 \%$ & 0.2 & 0.10 & 0.4 & 0.3 & 0 & $\%$ & $\%$ & $\%$ & & & & & & $0.16 \%$ \\
\hline & $1.80 \%$ & $1.75 \%$ & 1.0210 & 1.0070 & 2.0070 & & $2.38 \%$ & $2.21 \%$ & $2.20 \%$ & $7 \%$ & $1.18 \%$ & & & & $1.01 \%$ \\
\hline & $1.87 \%$ & $1.56 \%$ & $0.87 \%$ & $2.28 \%$ & $2.28 \%$ & & $2.12 \%$ & $1.87 \%$ & $1.82 \%$ & $1.63 \%$ & $1.66 \%$ & & & & $1.70 \%$ \\
\hline & & & & & & & & & & & & & & & \\
\hline & $6.38 \%$ & & $5.92 \%$ & & $6.40 \%$ & & & $5.87 \%$ & $5.79 \%$ & $4.98 \%$ & $5.38 \%$ & & & & $6.06 \%$ \\
\hline TC14.? & & & & & & & & $\%$ & & & & & & & \\
\hline & $8.57 \%$ & $8.56 \%$ & $8.85 \%$ & $7.71 \%$ & $7.17 \%$ & & $6.21 \%$ & $6.55 \%$ & $6.60 \%$ & $5.94 \%$ & $6.13 \%$ & & & & $8.15 \%$ \\
\hline TG46:2 & $3.47 \%$ & $3.71 \%$ & $3.34 \%$ & $4.57 \%$ & $4.80 \%$ & $4.22 \%$ & $4.67 \%$ & $4.66 \%$ & $4.62 \%$ & $3.23 \%$ & $3.69 \%$ & $3.05 \%$ & & & $3.77 \%$ \\
\hline & $1.09 \%$ & $1.09 \%$ & & & & & & & & & & & & & \\
\hline TG48:2 & $5.98 \%$ & $6.06 \%$ & $6.01 \%$ & $6.68 \%$ & $6.54 \%$ & $7.12 \%$ & $6.07 \%$ & $6.38 \%$ & $6.50 \%$ & $5.67 \%$ & $5.90 \%$ & $\%$ & & & $7.35 \%$ \\
\hline$: 3$ & 2.2 & 2.3 & & & & & & $\%$ & & & $\%$ & & & & $5 \%$ \\
\hline TG48:4 & $0.68 \%$ & $0.68 \%$ & $0.60 \%$ & $1.16 \%$ & $1.31 \%$ & $1.03 \%$ & $1.79 \%$ & $1.69 \%$ & $1.69 \%$ & $2 \%$ & $1.07 \%$ & & & & $0.70 \%$ \\
\hline TG50:2 & $8.78 \%$ & $8.80 \%$ & $9.52 \%$ & $7.28 \%$ & $6.75 \%$ & $8.23 \%$ & $6.03 \%$ & $6.44 \%$ & $6.48 \%$ & $7.35 \%$ & $6.87 \%$ & & & & $7.89 \%$ \\
\hline & 3 & 3 & 3.8 & 3 & $\%$ & & & 4. & 4. & 3. & 4. & & & & $7 \%$ \\
\hline TG50:4 & & $1.09 \%$ & $1.05 \%$ & & & & & $1.89 \%$ & $1.94 \%$ & & $1.41 \%$ & & & & $0.85 \%$ \\
\hline TGs & $16.72 \%$ & $16.68 \%$ & $18.89 \%$ & $12.74 \%$ & $10.96 \%$ & $14.73 \%$ & $10.41 \%$ & $9.82 \%$ & $9.91 \%$ & $8 \%$ & \#\#\#\#\# & $15.96 \%$ & $14.34 \%$ & $11.94 \%$ & $15.74 \%$ \\
\hline & $11.37 \%$ & $11.09 \%$ & $12.19 \%$ & & $8.52 \%$ & $10.05 \%$ & $7.97 \%$ & $8.67 \%$ & $8.08 \%$ & $10.53 \%$ & $9.27 \%$ & & & & $9.74 \%$ \\
\hline TG52:4 & $3.82 \%$ & $3.92 \%$ & $3.91 \%$ & $3.90 \%$ & $4.19 \%$ & $3.98 \%$ & $4.22 \%$ & $4.31 \%$ & $4.40 \%$ & $4.52 \%$ & $4.53 \%$ & $4.65 \%$ & $2.53 \%$ & $2.79 \%$ & $2.32 \%$ \\
\hline TG54:2 & & $3.87 \%$ & & & $3.76 \%$ & & & $3.02 \%$ & $3.10 \%$ & & $5.71 \%$ & & & & $T .20$ \\
\hline TG54:3 & $4.42 \%$ & $4.73 \%$ & $4.95 \%$ & $5.38 \%$ & $5.59 \%$ & $6.22 \%$ & $5.79 \%$ & $5.71 \%$ & $5.87 \%$ & $7.57 \%$ & $7.85 \%$ & $8.76 \%$ & $7.31 \%$ & $7.60 \%$ & $7.85 \%$ \\
\hline TG54:4 & $2.96 \%$ & $3.21 \%$ & $3.34 \%$ & $3.69 \%$ & $3.72 \%$ & $4.18 \%$ & $4.98 \%$ & $5.13 \%$ & $5.18 \%$ & $5.37 \%$ & $5.52 \%$ & $6.14 \%$ & $4.32 \%$ & $4.35 \%$ & $4.47 \%$ \\
\hline TG54:5 & $1.75 \%$ & $1.79 \%$ & $1.85 \%$ & $2.04 \%$ & $2.26 \%$ & $2.26 \%$ & $3.35 \%$ & $3.49 \%$ & $3.49 \%$ & $3.48 \%$ & $3.64 \%$ & $3.85 \%$ & $1.90 \%$ & $1.88 \%$ & $2.05 \%$ \\
\hline
\end{tabular}




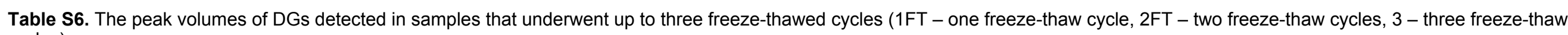
cycles)

\begin{tabular}{|c|c|c|c|c|c|c|c|c|c|c|c|c|c|c|c|}
\hline \multirow[b]{2}{*}{$\begin{array}{l}\text { Compoun } \\
\text { d }\end{array}$} & \multicolumn{3}{|c|}{ W13A } & \multicolumn{3}{|c|}{ W8 } & \multicolumn{3}{|c|}{ W18 } & \multicolumn{3}{|c|}{ W1B } & \multicolumn{3}{|c|}{ W4 } \\
\hline & $1 \mathrm{FT}$ & $2 \mathrm{FT}$ & $3 \mathrm{FT}$ & $1 \mathrm{FT}$ & $2 \mathrm{FT}$ & $3 \mathrm{FT}$ & $1 \mathrm{FT}$ & $2 \mathrm{FT}$ & $3 \mathrm{FT}$ & $1 \mathrm{FT}$ & $2 \mathrm{FT}$ & $3 \mathrm{FT}$ & $1 \mathrm{FT}$ & $2 \mathrm{FT}$ & $3 \mathrm{FT}$ \\
\hline & $9.25 \mathrm{E}+0$ & $1.86 \mathrm{E}+0$ & $3.52 \mathrm{E}+0$ & $3.44 \mathrm{E}+0$ & $1.17 \mathrm{E}+0$ & $8.35 \mathrm{E}+0$ & $2.94 \mathrm{E}+0$ & $3.59 \mathrm{E}+0$ & $4.06 \mathrm{E}+0$ & $1.21 \mathrm{E}+0$ & $3.28 \mathrm{E}+0$ & $3.77 \mathrm{E}+0$ & $1.45 \mathrm{E}+0$ & $3.86 \mathrm{E}+0$ & $3.26 \mathrm{E}+0$ \\
\hline \multirow[t]{2}{*}{ DG24:0 } & 5 & 6 & 6 & 6 & 7 & 6 & 6 & 6 & 6 & 6 & 6 & 6 & 6 & 6 & 6 \\
\hline & $1.78 \mathrm{E}+0$ & $4.52 \mathrm{E}+0$ & $8.88 \mathrm{E}+0$ & $5.63 E+0$ & $1.96 \mathrm{E}+0$ & 1.60E+0 & 4.97E+0 & $5.98 \mathrm{E}+0$ & $7.03 \mathrm{E}+0$ & $2.42 \mathrm{E}+0$ & $7.08 \mathrm{E}+0$ & $7.75 \mathrm{E}+0$ & $2.19 \mathrm{E}+0$ & $6.28 \mathrm{E}+0$ & $6.08 \mathrm{E}+0$ \\
\hline \multirow[t]{2}{*}{ DG 26:0 } & 6 & 6 & 6 & 6 & 7 & 7 & 6 & 6 & 6 & 6 & 6 & 6 & 6 & 6 & 6 \\
\hline & $2.19 E+0$ & $5.51 \mathrm{E}+0$ & $1.20 \mathrm{E}+0$ & $6.97 E+0$ & $2.50 \mathrm{E}+0$ & $2.32 \mathrm{E}+0$ & $5.92 \mathrm{E}+0$ & $6.26 \mathrm{E}+0$ & $8.74 \mathrm{E}+0$ & $3.23 E+0$ & $9.00 \mathrm{E}+0$ & $1.05 E+0$ & $2.57 \mathrm{E}+0$ & 7.57E+0 & $7.62 E+0$ \\
\hline \multirow[t]{2}{*}{ DG 28:0 } & 6 & 6 & 7 & 6 & 7 & 7 & 6 & 6 & 6 & 6 & 6 & 7 & 6 & 6 & 6 \\
\hline & $9.44 \mathrm{E}+0$ & $3.28 \mathrm{E}+0$ & $5.68 \mathrm{E}+0$ & $3.28 \mathrm{E}+0$ & $1.43 \mathrm{E}+0$ & $1.05 \mathrm{E}+0$ & $2.39 \mathrm{E}+0$ & $3.58 \mathrm{E}+0$ & $4.12 \mathrm{E}+0$ & $1.66 \mathrm{E}+0$ & $5.85 E+0$ & $5.98 \mathrm{E}+0$ & $1.44 \mathrm{E}+0$ & $5.06 \mathrm{E}+0$ & $4.60 \mathrm{E}+0$ \\
\hline \multirow[t]{2}{*}{ DG 28:1 } & 5 & 6 & 6 & 6 & 7 & 7 & 6 & 6 & 6 & 6 & 6 & 6 & 6 & 6 & 6 \\
\hline & $2.80 \mathrm{E}+0$ & $9.94 \mathrm{E}+0$ & $1.70 \mathrm{E}+0$ & $1.03 E+0$ & $4.92 E+0$ & $3.39 E+0$ & $1.13 E+0$ & $1.57 \mathrm{E}+0$ & $1.86 \mathrm{E}+0$ & $5.38 \mathrm{E}+0$ & $2.05 E+0$ & $2.11 \mathrm{E}+0$ & $4.26 \mathrm{E}+0$ & $1.44 \mathrm{E}+0$ & $1.26 \mathrm{E}+0$ \\
\hline \multirow[t]{2}{*}{ DG 28:2 } & 5 & 5 & 6 & 6 & 6 & 6 & 6 & 6 & 6 & 5 & 6 & 6 & 5 & 6 & 6 \\
\hline & $2.05 E+0$ & 4.77E+0 & $1.15 E+0$ & $4.99 \mathrm{E}+0$ & $1.75 E+0$ & $1.73 E+0$ & $4.46 \mathrm{E}+0$ & $5.29 \mathrm{E}+0$ & $5.95 E+0$ & $2.71 \mathrm{E}+0$ & $7.60 \mathrm{E}+0$ & $9.44 \mathrm{E}+0$ & $2.01 \mathrm{E}+0$ & $4.63 E+0$ & $5.12 E+0$ \\
\hline \multirow[t]{2}{*}{ DG30:0 } & 6 & 6 & 7 & 6 & 7 & 7 & 6 & 6 & 6 & 6 & 6 & 6 & 6 & 6 & 6 \\
\hline & $2.44 \mathrm{E}+0$ & $9.12 \mathrm{E}+0$ & $1.66 \mathrm{E}+0$ & 1.07E+0 & $4.60 \mathrm{E}+0$ & $4.00 \mathrm{E}+0$ & $7.33 \mathrm{E}+0$ & $1.12 \mathrm{E}+0$ & $1.49 \mathrm{E}+0$ & $4.42 \mathrm{E}+0$ & $1.76 \mathrm{E}+0$ & $1.89 E+0$ & $4.12 \mathrm{E}+0$ & $1.49 \mathrm{E}+0$ & $1.48 \mathrm{E}+0$ \\
\hline \multirow{2}{*}{ DG30:1 } & 6 & 6 & 7 & 7 & 7 & 7 & 6 & 7 & 7 & 6 & 7 & 7 & 6 & 7 & 7 \\
\hline & $7.57 \mathrm{E}+0$ & $3.18 \mathrm{E}+0$ & $5.92 \mathrm{E}+0$ & $3.93 E+0$ & $1.92 E+0$ & $1.55 \mathrm{E}+0$ & $3.61 \mathrm{E}+0$ & $6.09 \mathrm{E}+0$ & $7.28 \mathrm{E}+0$ & $1.57 \mathrm{E}+0$ & $6.56 \mathrm{E}+0$ & 7.17E+0 & 1.17E+0 & $4.89 \mathrm{E}+0$ & $4.82 E+0$ \\
\hline \multirow[t]{2}{*}{ DG30:2 } & 5 & 6 & 6 & 6 & 7 & 7 & 6 & 6 & 6 & 6 & 6 & 6 & 6 & 6 & 6 \\
\hline & $4.50 \mathrm{E}+0$ & $1.38 \mathrm{E}+0$ & $2.79 \mathrm{E}+0$ & $1.30 \mathrm{E}+0$ & $5.38 \mathrm{E}+0$ & 5.07E+0 & 1.16E+0 & $1.66 \mathrm{E}+0$ & $1.85 E+0$ & $7.61 \mathrm{E}+0$ & $2.44 \mathrm{E}+0$ & $3.01 \mathrm{E}+0$ & $4.82 \mathrm{E}+0$ & 1.49E+0 & $1.53 E+0$ \\
\hline \multirow[t]{2}{*}{ DG32:1 } & 6 & 7 & 7 & 7 & 7 & 7 & 7 & 7 & 7 & 6 & 7 & 7 & 6 & 7 & 7 \\
\hline & $1.48 \mathrm{E}+0$ & $5.22 \mathrm{E}+0$ & $1.11 \mathrm{E}+0$ & $5.16 \mathrm{E}+0$ & $2.44 \mathrm{E}+0$ & $2.16 \mathrm{E}+0$ & $5.98 \mathrm{E}+0$ & $9.42 \mathrm{E}+0$ & $1.05 E+0$ & $2.74 \mathrm{E}+0$ & $1.07 \mathrm{E}+0$ & 1.37E+0 & 1.67E+0 & $5.51 \mathrm{E}+0$ & $5.69 \mathrm{E}+0$ \\
\hline \multirow[t]{2}{*}{ DG32:2 } & 6 & 6 & 7 & 6 & 7 & 7 & 6 & 6 & 7 & 6 & 7 & 7 & 6 & 6 & 6 \\
\hline & $1.52 E+0$ & $4.41 \mathrm{E}+0$ & $8.65 E+0$ & $2.96 \mathrm{E}+0$ & $1.23 E+0$ & $1.21 \mathrm{E}+0$ & $2.25 \mathrm{E}+0$ & $3.31 \mathrm{E}+0$ & 3.37E+0 & $2.25 \mathrm{E}+0$ & $6.57 \mathrm{E}+0$ & $9.06 \mathrm{E}+0$ & $1.52 \mathrm{E}+0$ & $4.39 E+0$ & $4.11 \mathrm{E}+0$ \\
\hline \multirow[t]{2}{*}{ DG34:1 } & 7 & 7 & 7 & 7 & 8 & 8 & 7 & 7 & 7 & 7 & 7 & 7 & 7 & 7 & 7 \\
\hline & $5.82 E+0$ & $1.78 \mathrm{E}+0$ & $3.75 \mathrm{E}+0$ & 1.37E+0 & $5.74 \mathrm{E}+0$ & $5.79 E+0$ & $1.36 \mathrm{E}+0$ & $2.11 \mathrm{E}+0$ & $2.16 \mathrm{E}+0$ & $9.24 \mathrm{E}+0$ & $2.98 \mathrm{E}+0$ & $3.84 \mathrm{E}+0$ & $5.49 \mathrm{E}+0$ & $1.65 E+0$ & $1.57 \mathrm{E}+0$ \\
\hline \multirow[t]{2}{*}{ DG34:2 } & 6 & 7 & 7 & 7 & 7 & 7 & 7 & 7 & 7 & 6 & 7 & 7 & 6 & 7 & 7 \\
\hline & $1.08 \mathrm{E}+0$ & $3.91 \mathrm{E}+0$ & $8.51 \mathrm{E}+0$ & $3.26 \mathrm{E}+0$ & $1.48 \mathrm{E}+0$ & $1.44 \mathrm{E}+0$ & $3.19 E+0$ & $5.49 E+0$ & $5.48 \mathrm{E}+0$ & $2.33 E+0$ & $8.36 E+0$ & 1.12E+0 & $8.98 E+0$ & $3.17 \mathrm{E}+0$ & $3.09 E+0$ \\
\hline \multirow[t]{2}{*}{ DG34:3 } & 6 & 6 & 6 & 6 & 7 & 7 & 6 & 6 & 6 & 6 & 6 & 7 & 5 & 6 & 6 \\
\hline & $4.69 E+0$ & $1.52 \mathrm{E}+0$ & $2.74 \mathrm{E}+0$ & $1.04 \mathrm{E}+0$ & $4.56 \mathrm{E}+0$ & $4.70 \mathrm{E}+0$ & $1.13 E+0$ & $2.06 \mathrm{E}+0$ & $1.89 \mathrm{E}+0$ & $1.03 E+0$ & $3.12 \mathrm{E}+0$ & $4.13 E+0$ & $6.90 \mathrm{E}+0$ & $2.03 E+0$ & $1.93 E+0$ \\
\hline \multirow[t]{2}{*}{ DG36:2 } & 6 & 7 & 7 & 7 & 7 & 7 & 7 & 7 & 7 & 7 & 7 & 7 & 6 & 7 & 7 \\
\hline & $3.08 \mathrm{E}+0$ & $9.54 \mathrm{E}+0$ & 1.87E+0 & $7.61 \mathrm{E}+0$ & $3.61 \mathrm{E}+0$ & $3.59 E+0$ & $1.03 E+0$ & $1.78 \mathrm{E}+0$ & $1.79 E+0$ & $6.70 \mathrm{E}+0$ & $2.26 \mathrm{E}+0$ & $3.01 \mathrm{E}+0$ & $3.56 \mathrm{E}+0$ & $1.22 \mathrm{E}+0$ & $1.16 \mathrm{E}+0$ \\
\hline \multirow[t]{2}{*}{ DG36:3 } & 6 & 6 & 7 & 6 & 7 & 7 & 7 & 7 & 7 & 6 & 7 & 7 & 6 & 7 & 7 \\
\hline & $9.38 \mathrm{E}+0$ & $3.84 \mathrm{E}+0$ & $7.25 \mathrm{E}+0$ & $3.01 \mathrm{E}+0$ & $1.63 E+0$ & $1.48 \mathrm{E}+0$ & $4.49 E+0$ & $8.49 E+0$ & $8.12 E+0$ & $2.96 \mathrm{E}+0$ & $1.06 \mathrm{E}+0$ & $1.34 \mathrm{E}+0$ & $1.01 \mathrm{E}+0$ & $3.62 E+0$ & $3.52 E+0$ \\
\hline DG36:4 & 5 & 6 & 6 & 6 & 7 & 7 & 6 & 6 & 6 & 6 & 7 & 7 & 6 & 6 & 6 \\
\hline
\end{tabular}


Table S7. The \%RSD values calculated for phospholipid relative amounts in samples that underwent three freeze-thawed cycles

\begin{tabular}{lccccc}
\hline & W13A & W8 & W18 & W1B & W4 \\
\hline $\begin{array}{l}\text { LysoPC16:0 } \\
\text { Lyso- }\end{array}$ & $18 \%$ & $3 \%$ & $9 \%$ & $9 \%$ & $5 \%$ \\
$\begin{array}{l}\text { PC18:1 } \\
\text { Lyso- }\end{array}$ & $4 \%$ & $5 \%$ & $5 \%$ & $6 \%$ & $3 \%$ \\
PC18:2 & $11 \%$ & $3 \%$ & $6 \%$ & $3 \%$ & $4 \%$ \\
Lyso- & & & & & \\
PE18:1 & $12 \%$ & $11 \%$ & $7 \%$ & $3 \%$ & $18 \%$ \\
LYsoPE18:2 & $5 \%$ & $6 \%$ & $5 \%$ & $12 \%$ & $14 \%$ \\
PC32:0 & $11 \%$ & $10 \%$ & $7 \%$ & $13 \%$ & $4 \%$ \\
PC34:1 & $5 \%$ & $2 \%$ & $1 \%$ & $1 \%$ & $2 \%$ \\
PC34:2 & $2 \%$ & $4 \%$ & $1 \%$ & $5 \%$ & $1 \%$ \\
PC36:2 & $3 \%$ & $2 \%$ & $2 \%$ & $2 \%$ & $1 \%$ \\
PE34:1 & $10 \%$ & $7 \%$ & $4 \%$ & $2 \%$ & $6 \%$ \\
PE34:2 & $5 \%$ & $5 \%$ & $8 \%$ & $3 \%$ & $7 \%$ \\
PE36:1 & $7 \%$ & $3 \%$ & $3 \%$ & $10 \%$ & $11 \%$ \\
PE36:2 & $2 \%$ & $1 \%$ & $1 \%$ & $2 \%$ & $4 \%$ \\
SMd34:1 & $2 \%$ & $4 \%$ & $4 \%$ & $3 \%$ & $2 \%$ \\
SMd36:1 & $4 \%$ & $3 \%$ & $2 \%$ & $3 \%$ & $0 \%$ \\
SMd38:1 & $2 \%$ & $5 \%$ & $2 \%$ & $2 \%$ & $3 \%$ \\
SMd40:1 & $2 \%$ & $3 \%$ & $3 \%$ & $4 \%$ & $3 \%$ \\
SMd42:1 & $5 \%$ & $7 \%$ & $3 \%$ & $6 \%$ & $5 \%$ \\
\hline
\end{tabular}

